\title{
Long-Lasting Cognitive Deficits Resulting from Adolescent Nicotine Exposure in Rats
}

\author{
Danielle S Counotte', Sabine Spijker', Linda H Van de Burgwal', François Hogenboom², \\ Anton NM Schoffelmeer', Taco J De Vries ${ }^{1,2}$, August B Smit' and Tommy Pattij*,2 \\ 'Department of Molecular and Cellular Neurobiology, Center for Neurogenomics and Cognitive Research, Vrije Universiteit, Amsterdam, \\ The Netherlands; '2Department of Anatomy and Neurosciences, Center for Neurogenomics and Cognitive Research, VU University Medical \\ Center, Amsterdam, The Netherlands
}

\begin{abstract}
Adolescence is a developmental period, during which the brain and particularly medial prefrontal cortical (mPFC) regions thereof have not fully matured. Because epidemiological data have suggested that adolescent nicotine use may result in disturbances in cognitive function in adulthood, we investigated the long-term effects of adolescent nicotine exposure in rats. Male Wistar rats were exposed to either nicotine (three times daily, $0.4 \mathrm{mg} / \mathrm{kg}$ s.c.) or saline for 10 days during (postnatal day (PND) 34-43) or following (PND 60-69) adolescence. After 5 weeks during adulthood, separate groups of animals were tested in operant paradigms taxing attention and distinct measures of impulsivity. Visuospatial attention and impulsive action were tested in the five-choice serial reaction time task, whereas impulsive choice was assessed in the delayed reward task. Our data show that adolescent, but not postadolescent, nicotine exposure affects cognitive performance in adulthood and results in diminished attentional performance and increments in impulsive action, while leaving impulsive choice intact. This altered cognitive performance appeared to be associated with enhanced releasability of dopamine in the mPFC. Together, these data suggest that adolescence is a time window during which the brain is vulnerable to long-lasting cognitive disturbances resulting from nicotine exposure.

Neuropsychopharmacology (2009) 34, 299-306; doi:I0.1038/npp.2008.96; published online 25 June 2008
\end{abstract}

Keywords: adolescence; nicotine; attention; impulsivity; prefrontal cortex

\section{INTRODUCTION}

Adolescence is a critical developmental period during which the human brain continues to develop until approximately the age of 20 , with synaptic pruning and myelination of frontal cortical regions maturing in the later stages of adolescence (for review, see Casey et al, 2005). Because the adolescent brain is still plastic, exposure to addictive substances such as nicotine inhaled from tobacco smoke may greatly impact the development of frontal cortical regions and thus lead to changes in brain function that persist in adulthood (for review, see Slotkin, 2004). It is well established that frontostriatal brain regions are essential in executive functions, such as attentional processing and inhibitory response control as well as decision-making processes (for reviews, see Arnsten and Li, 2005; Miller and Cohen, 2001). The late development of these brain regions involved in impulse control might cause adolescents to be

\footnotetext{
*Correspondence: Dr T Pattij, Department of Anatomy and Neurosciences, Center for Neurogenomics and Cognitive Research, VU University Medical Center, Van der Boechorststraat 7, Amsterdam I08I BT, Netherlands, Tel: + 3120444 8089, E-mail: t.pattij@vumc.nl Received 18 February 2008; revised 23 May 2008; accepted 23 May 2008
}

more risk-taking and to experiment with drugs, such as nicotine. Indeed, most adult smokers start their habit before the age of 19 and several retrospective and prospective studies have suggested that early onset of smoking behavior may predict cigarette smoking and nicotine dependence in adulthood (Brown et al, 1996; Chassin et al, 1996; Dappen et al, 1996). In the Netherlands, $71 \%$ of adolescents have tried a cigarette at least once (Van Andel et al, 2003), and approximately $40 \%$ smoke at least monthly at the age of 19 . These figures are comparable to other European countries (Godeau et al, 2004), and even higher compared to the United States, where $25 \%$ of adolescents report cigarette use in the past 30 days (Johnson et al, 2006). Together, these numbers stress the importance to study the potentially hazardous long-term effects of nicotine on the developing adolescent brain.

To date, clinical studies assessing changes in cognitive functioning following nicotine exposure in adolescents have largely focused on its short-term effects. Moreover, in these studies, levels of smoking were often determined by selfreport measures and did not control for presmoking levels of cognitive performance. Nevertheless, in adolescent smokers disturbances in working memory processes and attention have been reported (Jacobsen et al, 2005) as well as reduced attention-associated prefrontal cortical 
blood-oxygen level dependent responses (Musso et al, 2007). Furthermore, epidemiological data suggest longlasting behavioral disturbances and mental health problems in adulthood following adolescent nicotine use (for review, see Mathers et al, 2006). However, the difficulty to control gene-environment interactions in the human population hampers a straightforward interpretation of adolescent nicotine exposure on subsequent behavioral changes.

Animal models have greatly contributed to our understanding of the adolescent brain and are well suited to explore putative causal relationships between early drug exposure during adolescence and cognitive functioning (in the absence of nicotine) in adulthood. In rats, typical adolescent-like behaviors include increments in peerdirected behavior, risk-taking behavior, indexed by elevated exploratory behavior and novelty-seeking, and altered sensitivity to drugs of abuse. These behaviors are generally observed in the period that ranges from approximately postnatal day (PND) 28-50 (for review, see Spear, 2000).

The long-term effects of adolescent nicotine exposure on cognitive performance in rats have not been studied before. Previous work in rats has shown that adolescent nicotine, followed by $2-5$ weeks of abstinence, has long-term effects on behavior in both the emotional and motivational domain, exemplified by an increase in novelty-induced activity, elevated anxiety, higher levels of passive avoidance, and enhanced vulnerability to nicotine self-administration (Adriani et al, 2006, 2003; Slawecki et al, 2005; Trauth et al, 2000).

The aim of the present study was to assess long-term effects of nicotine exposure specifically during the adolescent period on executive cognitive functions in rats. To this end, different behavioral paradigms were used to assess visuospatial attention as well as distinct forms of impulsivity, namely the 5-choice serial reaction time task (5-CSRTT) and the delayed reward paradigm that measure aspects of impulsive action and impulsive choice, respectively (for recent review, see Winstanley et al, 2006a). Whereas acute and (sub)chronic nicotine challenges have been shown to increase impulsivity and might result in improved visuospatial attention in the 5-CSRTT (Day et al, 2007; Grottick and Higgins, 2000; Hahn et al, 2002; Van Gaalen et al, 2006a), the present study focused on the long-term effects of adolescent nicotine exposure on 5-CSRTT performance in adulthood. Moreover, to explore putative long-lasting neuroplasticity in frontostriatal brain regions resulting from adolescent nicotine exposure, we also measured the electrically evoked release of (radiolabeled) dopamine in slices of the medial prefrontal cortex (mPFC) and nucleus accumbens $(\mathrm{Acb})$ in separate groups of rats.

\section{MATERIALS AND METHODS}

\section{Animals}

Timed pregnant Wistar female rats arrived at 5 days of gestation (Harlan, Horst, the Netherlands) and were housed individually in Macrolon cages under standard conditions and a reversed day-night cycle (lights on from 1900 to 0700 hours). Upon delivery, litters were culled to eight pups per mother and preferably consisted of male rats only, but were occasionally matched with female rats. At PND 21, animals were weaned and housed two per cage. Only male rats were used in these experiments. During the course of behavioral testing, animals were food restricted to maintain $85-90 \%$ of their free-feeding weight. Water was available ad libitum. All experiments were approved by the animal ethical committee of the Vrije Universiteit, Amsterdam, The Netherlands.

\section{Nicotine Exposure}

Two groups of 48 animals and a third group of 32 rats were injected subcutaneously with either nicotine $(0.4 \mathrm{mg} / \mathrm{kg}$, calculated as a base $((-)$ Nicotine hydrogen tartrate salt; Sigma, St Louis, MO, USA)) or saline three times a day (at 1000,1300 , and 1500 hours) for 10 days. Nicotine was administered to half of the animals during adolescence (PND 34-43), whereas the other animals were exposed during adulthood (PND 60-69). The saline controls were littermates of the nicotine-exposed animals in both age groups. Treatment of adult and adolescent animals occurred simultaneously and 5 weeks later, when groups had reached adulthood, behavioral tests commenced ( $n=12$ per group). The first group of 48 animals was trained in the 5-CSRTT, and the second group in the delayed reward task (DRT). A third group of rats was decapitated 5 weeks following nicotine exposure and was used to study neurotransmitter release ( $n=8$ per group). All behavioral testing was carried out in the dark period under red light conditions.

\section{5-CSRTT}

Experiments were conducted in rat operant chambers with stainless steel grid floors (MED-NPW-5L; Med Associates Inc., St Albans, VT, USA) as described elsewhere (Van Gaalen et al, 2006a). On-line control of all operant chambers and data collection were performed using MED-PC version IV (Med Associates Inc.). Five sessions were scheduled per week from Monday until Friday, one session per day.

A more detailed description of training in the 5-CSRTT has been reported previously (Van Gaalen et al, 2006a). In short, rats were trained to detect and respond to a $1 \mathrm{~s}$ visual stimulus in either one of five apertures, during presentation of the stimulus or during the $2 \mathrm{~s}$ limited hold period, to obtain a food reward $(45 \mathrm{mg}$, Formula P; Research Diets Inc., New Brunswick, NJ, USA). Each session terminated after 100 trials or $30 \mathrm{~min}$, whichever occurred first. Initially the duration of this stimulus was $32 \mathrm{~s}$ and was gradually decreased to $1 \mathrm{~s}$ over sessions until animals reached stable baseline performance (accuracy $>80 \%$ correct choice and $<20 \%$ errors of omission). Incorrect, premature responses (responses during the $5 \mathrm{~s}$ intertrial interval (ITI)) and errors of omission did not lead to the delivery of a food reward and resulted in a $5 \mathrm{~s}$ time-out period during which the house light was extinguished. Responses during the time-out period resulted in a new time-out period. Perseverative responses, ie repeated responding into an aperture following correct choice and before pellet collection were measured but did not have any programmed consequences. The following behavioral measures were recorded: (1) accuracy, ((number correct trials/(correct + incorrect trials)) $\times 100)$; (2) latency of correct responses; (3) percentage of premature responses ((mean number premature 
responses during the ITI/mean number (correct + incorrect + perseverative + premature responses) $\times 100$ ); (4) perseverative responses after correct choice; (5) percentage of omissions ((number omissions/(correct + incorrect responses + omissions) $) \times 100)$, and (6) feeder latency.

During acquisition one animal from the adolescent nicotine group unexpectedly died, whereas one animal from the adolescent saline group persistently displayed high levels of omissions $(>60 \%)$. Therefore these animals were excluded from all analyses.

\section{Delayed Reward}

Experiments were conducted in the same operant chambers as described for the 5-CSRTT. A more detailed description of training in the DRT has been previously reported (Van Gaalen et al, 2006b). In short, rats needed to start a trial by nose poking in the middle illuminated aperture (initiation period). This dimmed the cue light and illuminated the apertures immediately adjacent to the middle aperture (choice phase). When a rat failed to respond within the initiation period, the cue light was extinguished, and an ITI started. The choice phase lasted $10 \mathrm{~s}$ or until a nose poke was made. The latter resulted in the delivery of either a small reward (one pellet) or large reward (four pellets) depending on the aperture. The aperture associated with small and large reward was always fixed for each animal, but counterbalanced across rats. After the choice phase, an ITI started until the next trial started. Nose poking in any of the nonilluminated apertures was recorded, but had no consequences. A session was divided into five blocks of 12 trials, each starting with two forced trials, during which, after initiating the trial either the aperture associated with small or large reward was illuminated in a counterbalanced fashion. For the next 10 trials, the animal had a free choice. The delay for the large reward increased per block of 10 trials from $0,2,4,8,12 \mathrm{~s}$ to eventually $0,5,10,20,40 \mathrm{~s}$.

\section{Neurotransmitter Release}

An additional cohort of 32 rats was exposed to nicotine or saline as described above. After 5 weeks, comparable to the time window of training in the behavioral paradigms, animals were decapitated and the Acb and mPFC (infralimbic and prelimbic cortex) were rapidly dissected from the brain. In replicate experiments, brain tissue was sliced $(0.3 \times 0.3 \times 2 \mathrm{~mm})$ and for each brain region the slices of two animals were pooled. Subsequently, brain slices were incubated and superfused as described before (Schoffelmeer et al, 1988). Briefly, slices were incubated for $15 \mathrm{~min}$ in Krebs-Ringer bicarbonate containing $5 \mu \mathrm{Ci}\left[{ }^{3} \mathrm{H}\right]$ dopamine (Radiochemical Centre, Amersham, Buckinghamshire, UK) in an atmosphere of $95 \% \mathrm{O}_{2}-5 \% \mathrm{CO}_{2}$ at $37^{\circ} \mathrm{C}$. As the $\mathrm{Acb}$ and $\mathrm{mPFC}$ have a dense noradrenergic innervation, $3 \mu \mathrm{M}$ desipramine (Sigma) was added to the medium of these brain structures to prevent accumulation of $\left[{ }^{3} \mathrm{H}\right]$ dopamine in noradrenergic nerve terminals. After labeling, slices were rapidly washed and transferred to each of the 24 chambers of a superfusion apparatus (approximately $4 \mathrm{mg}$ tissue in $0.2 \mathrm{ml}$ volume $)$ and superfused $(0.2 \mathrm{ml} / \mathrm{min})$ with medium gassed with $95 \% \mathrm{O}_{2}-5 \% \mathrm{CO}_{2}$ at $37^{\circ} \mathrm{C}$. In each experiment, neurotransmitter release from brain slices of all four groups (nicotine and saline pretreatment during or following adolescence) was studied simultaneously in 24 parallel superfusion chambers. The superfusate was collected as 10 -min samples after $40 \mathrm{~min}$ of superfusion ( $t=40 \mathrm{~min}$ ). Neurotransmitter release was induced by exposing the slices to electrical biphasic block pulses (Acb: $1 \mathrm{~Hz}, 2 \mathrm{~ms}$ at $30 \mathrm{~mA}$, mPFC: $1 \mathrm{~Hz}, 2 \mathrm{~ms}$ at $20 \mathrm{~mA}$, for $10 \mathrm{~min}$ at $t=50 \mathrm{~min}$. The radioactivity remaining at the end of the experiment was extracted from the tissue with $0.1 \mathrm{~N} \mathrm{HCl}$. The radioactivity in superfusion fractions and tissue extracts was determined by liquid scintillation counting. The efflux of radioactivity during each collection was expressed as percentage of the amount of radioactivity in the slices at the beginning of the respective collection period. The electrically evoked release of neurotransmitter was calculated by subtracting the spontaneous efflux of radioactivity from the total overflow of radioactivity during stimulation and the next $10 \mathrm{~min}$. A linear decline from the 10 -min interval before that 20-30 min after the start of stimulation was assumed for calculation of the spontaneous efflux of radioactivity. The release evoked was expressed as percentage of the content of radioactivity of the slices at the start of the stimulation period.

\section{Statistical Analyses}

Data obtained in the 5-CSRTT were subjected to repeated measures analysis of variance (ANOVA) with exposure regimen (age and drug) as between-subjects variables and session as within-subjects variables using the Statistical Package for the Social Sciences version 11 (SPSS Inc., Chicago, IL, USA). Data obtained in the DRT were subjected to repeated measures ANOVA with exposure regimen (age and drug) as between-subjects variables and delay to large reinforcer (DRT) as within-subjects variables. Neurotransmitter release in pooled brain structures from nicotine treated rats was calculated as percentage change in neurotransmitter release from saline-treated rats and analyzed using univariate ANOVA with exposure regimen (age and drug) as between-subjects variable. The homogeneity of variance across groups was determined using Mauchly's tests for equal variances and in case of violation of homogeneity, corrected and therefore more conservative HuynhFeldt probability values were used for subsequent analyses. In case of statistically significant main effects, further post hoc comparisons were conducted using Student-NewmanKeuls Tests. The level of probability for statistically significant effects was set at 0.05 .

\section{RESULTS}

\section{Baseline Performance in the 5-CSRTT}

Stable baseline performance, during which rats always completed all 100 trials, was reached in the four different groups within 28-30 sessions after the start of training. Under these conditions, the groups differed in terms of accuracy and premature responding. They did not show differences in acquisition of the task; differences became apparent after nine sessions on a stimulus duration of $1 \mathrm{~s}$. As displayed in Figures $1 \mathrm{a}$ and $\mathrm{b}$, under stable baseline responding over five consecutive sessions (session: 

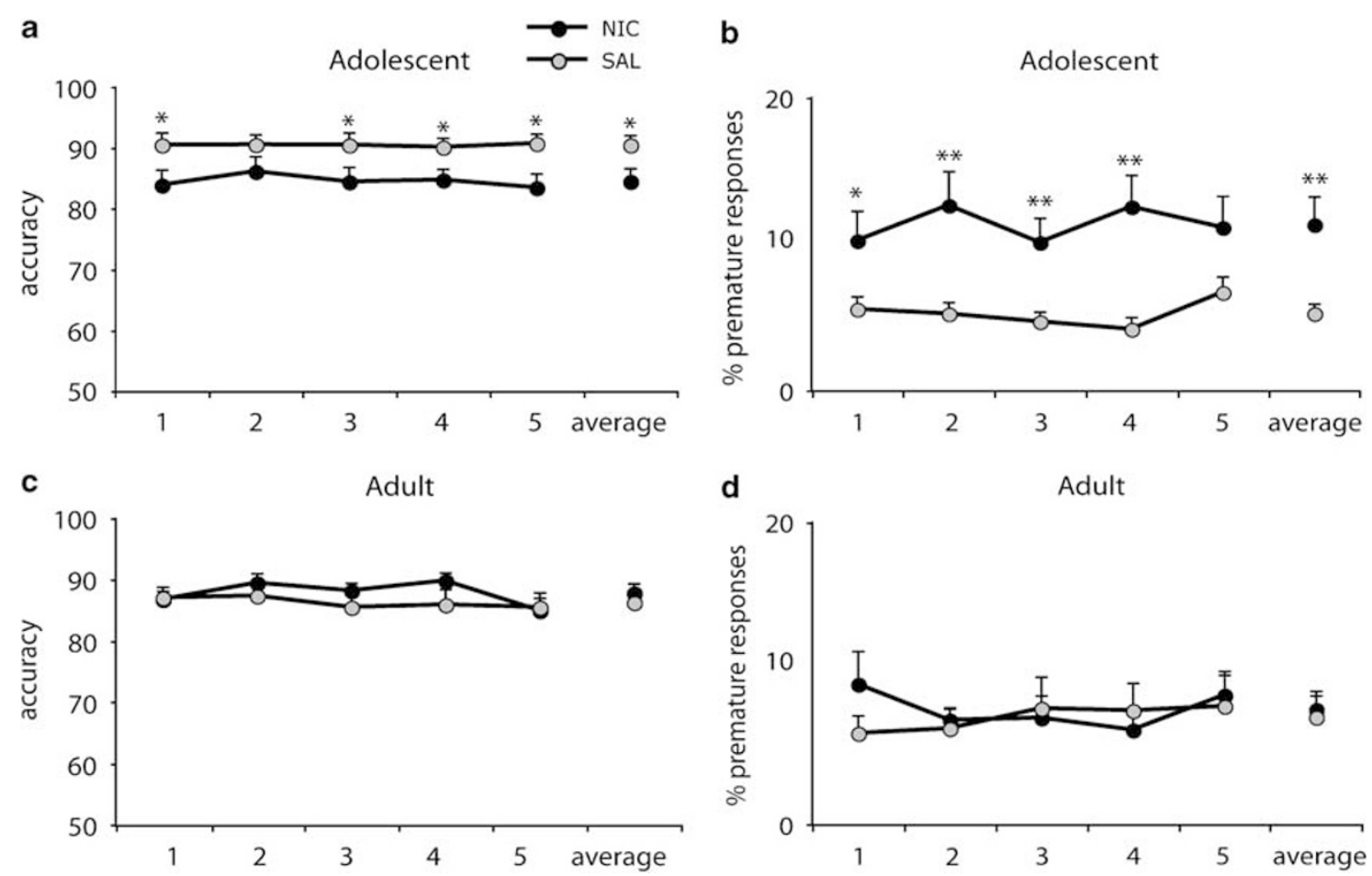

Figure I Performance in the five-choice serial reaction time task (5-CSRTT) over five baseline sessions, including the average of these five sessions, Accuracy $(a, c)$ calculated as the percentage of correct responses over total correct and incorrect responses. Impulsive action (b, d) calculated as the percentage of responses during the intertrial interval (ITI) (premature responses) over total responses (correct, incorrect, premature, and perseverative responses). Depicted are means \pm SEM of nicotine- $(-)$ and saline $(O)$-pretreated adolescent $(a, b)$ and adult $(c, d)$ animals. * $p<0.05$ compared to nicotine-pretreated rats and $* * * 0.01$ compared to saline-pretreated rats.

Table I Behavioral Parameters in the 5-CSRTT Averaged over five Baseline Sessions

\begin{tabular}{|c|c|c|c|c|}
\hline \multirow{2}{*}{ 5-CSRTT parameter } & \multicolumn{2}{|c|}{ Adolescent } & \multicolumn{2}{|c|}{ Adult } \\
\hline & Saline & Nicotine & Saline & Nicotine \\
\hline Correct responses & $84.2 \pm 2.3$ & $68.4 \pm 3.5 *$ & $74.5 \pm 2.9$ & $77.3 \pm 2.6$ \\
\hline Incorrect responses & $8.0 \pm 1.4$ & $12.9 \pm 1.9$ & $12.0 \pm 1.7$ & $10.6 \pm 1.3$ \\
\hline$\%$ Omissions & $8.7 \pm 1.9$ & $14.8 \pm 3.4$ & $13.0 \pm 2.9$ & $11.0 \pm 2.3$ \\
\hline Premature responses & $5.8 \pm 0.9$ & $14.2 \pm 3.8 *$ & $8.1 \pm 2.1$ & $8.3 \pm 1.3$ \\
\hline Perseverative responses & $9.8 \pm 2.2$ & $5.1 \pm 1.0$ & $5.5 \pm 1.0$ & $7.6 \pm 1.5$ \\
\hline Time-out responses & $21.2 \pm 2.7$ & $43.0 \pm 4.7$ *** & $33.2 \pm 4.0$ & $29.6 \pm 3.6$ \\
\hline Correct response latency (s) & $0.30 \pm 0.01$ & $0.33 \pm 0.02$ & $0.33 \pm 0.01$ & $0.32 \pm 0.0$ \\
\hline Feeder latency (s) & $1.17 \pm 0.16$ & $0.93 \pm 0.07$ & $1.16 \pm 0.19$ & $1.06 \pm 0.0$ \\
\hline
\end{tabular}

Abbreviation: 5-CSRTT, 5-choice serial reaction time task.

Mean \pm SEM is depicted. $* p<0.05$ and ${ }^{*} * p<0.005$ compared to their saline control.

$\mathrm{F}(1,42)=2.23$, NS), previous nicotine exposure differentially affected accuracy in adulthood depending on the age of exposure (age: $\mathrm{F}<1$; drug: $\mathrm{F}(1,42)=2.32$, NS; age $\times$ drug: $\mathrm{F}(1,42)=5.98, p=0.02)$. In particular, nicotine exposure during, but not following adolescence had long-term detrimental effects on accuracy of performance. In-depth analyses indicated that reduced accuracy in the adolescent nicotine group resulted from a reduction in correct detection of the stimulus, rather than from an increase in incorrect responses (Table 1).
In addition to changes in visuospatial attentional performance resulting from adolescent nicotine exposure, impulsive action (measured as the percentage of premature responses, ie responses during the ITI) was also differentially affected by nicotine when administered during adolescence versus adulthood (Figures $1 \mathrm{c}$ and d; session and age: both $\mathrm{Fs}<1$; drug: $\mathrm{F}(1,42)=5.77, p=0.02$; age $\times$ drug: $\mathrm{F}(1,42)=4.09, p=0.049)$. Further comparisons revealed a twofold increase in the percentage of premature responding when animals were exposed to nicotine during adolescence. In contrast, nicotine exposure during adulthood had no long-term effects on premature responding.

Other behavioral parameters in the 5-CSRTT (depicted in Table 1), including the percentage of omissions, correct response latencies, and feeder latencies were neither changed by periadolescent nor adult exposure with nicotine, indicating that there were no differences in motivation or motor activity. Nonetheless, in addition to impulsive action, there was an increase in time-out responses following adolescent nicotine exposure (age: $\mathrm{F}<1$; drug: $\mathrm{F}(1,42)=$ $5.68, p=0.02$; age $\times$ drug: $\mathrm{F}(1,42)=11.05, p=0.002)$.

\section{Performance in the DRT}

To study whether adolescent nicotine exposure would increase other forms of impulsivity, a different group of animals was trained in the DRT measuring impulsive choice. Stable baseline responding was achieved in the DRT after approximately 27 sessions, with no differences in acquisition between periadolescent and adult rats exposed to nicotine. As shown in Figure 2, in all groups the choice 


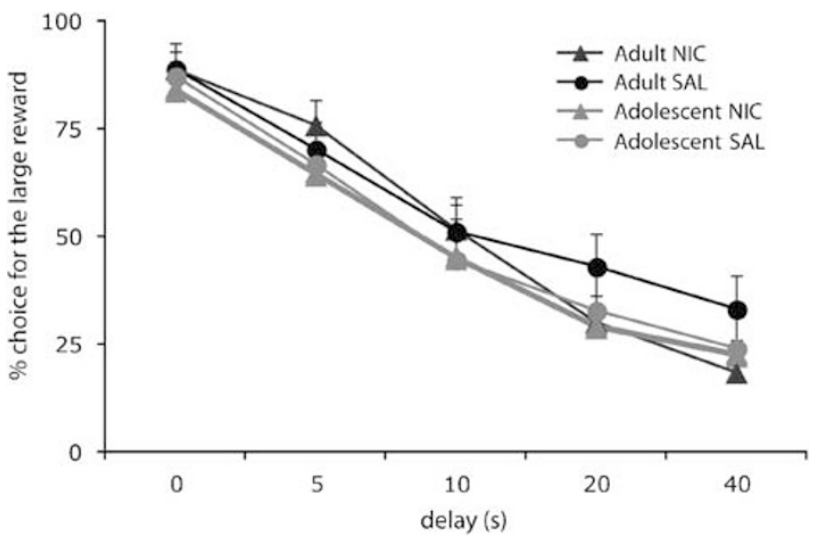

Figure 2 The effects of nicotine pretreatment on the delayed reward task. Depicted is the mean \pm SEM percentage choice for the large reinforcer as a function of delay, calculated over five baseline sessions.

for the large reinforcer significantly declined with increasing delays, indicating that all groups showed a hyperbolic delay discounting curve (delay: $\mathrm{F}(1,44)=193.19, p<0.001$ ). Moreover, preference for the large reinforcer as a function of the delay was not differentially affected by periadolescent or adult nicotine exposure (delay $\times$ age: $\mathrm{F}<1$; delay $\times$ drug: $\mathrm{F}(1,44)=1.60$, NS; delay $\times$ age $\times$ drug: $\mathrm{F}(1,44)=1.09$, NS). Again, there were no differences in motivation between the groups, indicated by similar numbers of choice omissions and omissions to start a trial (data not shown).

\section{In Vitro Neurotransmitter Release}

The electrically evoked ${ }^{3} \mathrm{H}$-dopamine release from $\mathrm{mPFC}$ slices in saline-treated animals amounted to $6.4 \pm 0.3 \%$ of total tissue tritium for adolescent and $7.5 \pm 0.8 \%$ of total tissue tritium for adult nicotine-exposed animals. As shown in Figure 3a, nicotine exposure differentially affected evoked ${ }^{3} \mathrm{H}$-dopamine release in adulthood. In periadolescent nicotine-exposed animals, electrically evoked ${ }^{3} \mathrm{H}-$ dopamine release was increased approximately 1.4-fold, whereas in adult nicotine-exposed animals electrically evoked ${ }^{3} \mathrm{H}$-dopamine release was not changed in this brain region (age: $\mathrm{F}(1,15)=1.30$, NS; drug: $\mathrm{F}<1$; age $\times$ drug: $\mathrm{F}(1,15)=12.70, p=0.004)$. The electrically evoked ${ }^{3} \mathrm{H}-$ dopamine release from Acb slices in saline-treated animals amounted to $3.4 \pm 0.1 \%$ of total tissue tritium for adolescent and $3.1 \pm 0.2 \%$ of total tissue tritium for adult-exposed animals. No long-term changes were found in electrically evoked ${ }^{3} \mathrm{H}$-dopamine release in the Acb as a result of periadolescent or adult nicotine exposure (Figure $3 \mathrm{~b}$; age: $\mathrm{F}(1,15)=1.03$, NS; drug and age $\times$ drug: both $\mathrm{F}$ 's $<1)$.

\section{DISCUSSION}

The present findings demonstrate that periadolescent, but not postadolescent nicotine exposure, has long-lasting effects on cognitive functioning in rats. Specifically, the present data point to deficits in visuospatial attention and increased impulsivity that is prominent after more than 5 weeks of abstinence. Deficits in impulse control were observed on impulsive action as measured in the 5-CSRTT,

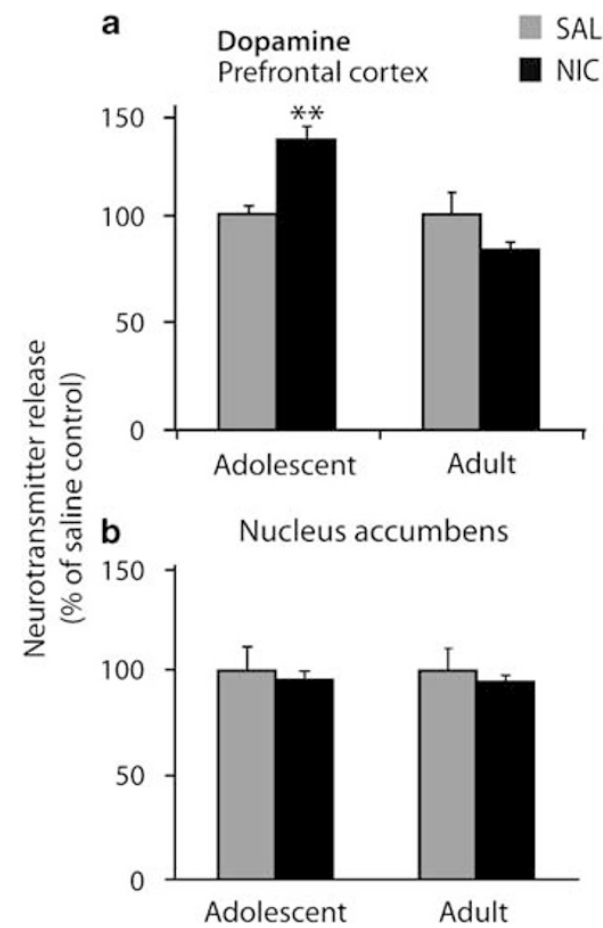

Figure 3 Electrically evoked dopamine release. Dopamine release in the medial prefrontal cortex (a) and nucleus accumbens (b) of animals pretreated with nicotine $(\mathbf{a})$ or saline $(\square)$ during adolescence or adulthood. Data of nicotine-pretreated animals are expressed as the percentage of saline-pretreated animals. Depicted are means \pm SEM of four experiments. ${ }^{*} * 0.005$ compared to their respective saline controls.

but not on a different form of impulsivity, ie impulsive choice as measured in a delayed reward paradigm. In separate groups of animals, neurochemical analyses demonstrated long-term hyperresponsiveness of dopaminergic nerve terminals in the mPFC following exposure to nicotine during, but not following, adolescence. Overall, these data suggest that adolescence is a unique time window during which the brain is more vulnerable and prone to develop long-lasting changes in cognitive functions resulting from nicotine exposure.

\section{Adolescent Nicotine Exposure and Impulsivity}

Smoking appears to be closely related to several forms of impulsivity in humans. For example, adult smoking has been associated with both impulsive choice (Bickel et al, 1999; Mitchell, 1999) as well as deficits in inhibitory control when compared to nonsmokers (Mitchell, 1999; Skinner et al, 2004; Spinella, 2002). However, whether impulsivity in adult smokers results from nicotine exposure, or alternatively, is a preexisting vulnerability trait predisposing individuals to initiate and maintain smoking is as yet unclear. Recent preclinical data would support the latter view and suggest that trait impulsivity may predict the vulnerability to initiate and maintain nicotine seeking (Diergaarde et al, 2008).

To date, human evidence describing the long-term effects (in absence of nicotine) of adolescent smoking on impulsivity in adulthood is limited. It has been demonstrated recently though, that the inability to abstain from smoking in adolescents is associated with elevated levels of impulsive 
action in the continuous performance task as well as increased impulsive choice in a real-time delay discounting paradigm (Krishnan-Sarin et al, 2007), which is comparable to our rat delayed reward paradigm. Nevertheless, the longlasting effects of smoking on measures of impulsivity in both abstinent as well as nonabstinent adolescent smokers and nonsmoking adolescents have not been evaluated as yet. Similarly, adolescent smokers show higher rates of delay discounting compared to nonsmokers. However, reported length of smoking history did not correlate with delay discounting, suggesting that adolescent nicotine exposure has no long-term effects on delay discounting, which is comparable to our findings (Reynolds, 2004).

\section{Adolescent Nicotine Exposure and Attention}

Another important observation in the present study is that exposure to nicotine during, but not following adolescence, causes long-term decrements in attentional performance. These disturbances in cognitive performance appear not to be caused by deficits in motivation and motor behavior, as other behavioral parameters in the 5-CSRTT, such as percentage omissions, feeder latencies, and correct response latencies are not affected by adolescent nicotine treatment. Interestingly, previous studies have reported an inverse correlation between attentional performance and premature responding in the 5-CSRTT (Dalley et al, 2008; Puumala et al, 1996). Therefore, the observed deficits in attentional performance and impulsive action might be associated and derived from a similar neurobiological construct.

Clinical evidence for long-term effects of adolescent smoking on executive functions such as attentional performance is scarce. However, our findings are in line with recent observations indicating short-term impairments in auditory attention in adolescent male smokers (Jacobsen et al, 2005, 2007). In addition, in a longitudinal study it was shown that similar cognitive deficits in adolescent smokers persist after controlling for presmoking levels of cognitive performance (Fried et al, 2006). Most of these deficits showed reversal after cessation of smoking, suggesting that they were transient and/or associated with nicotine withdrawal. In this latter study, decrements on performance on an arithmetic task persisted, indicating that adolescent nicotine exposure has some specific, longer-lasting effects in humans. The present data further extend these observations by demonstrating long-lasting disturbances on visuospatial attention that persist into adulthood.

\section{Adolescent Nicotine Exposure and Dopamine Release}

In terms of neurobiological changes, to date only few studies have addressed the long-term effects of nicotine exposure during adolescence. A recent study showed that adolescent nicotine exposure causes cortical cholinergic hypoactivity, which persists into adulthood (Slotkin et al, 2007). Our neurochemical data show long-lasting increments in depolarization-induced dopamine release in slices of the MPFC, but not of the Acb, in animals exposed to nicotine during, but not following, adolescence. Whether these changes in dopamine releasability are causally related to the observed decrements in visuospatial attention and impulsive action remains to be established. However, dopamine transmission has been shown to be involved in modulating attentional performance in the 5-CSRTT. For instance, several studies have demonstrated that under similar test conditions amphetamine impairs visuospatial attention (Cole and Robbins, 1987; Harrison et al, 1997; Pattij et al, 2007), an effect that could be mimicked by the selective dopamine reuptake inhibitor GBR 12909 (Van Gaalen et al, 2006a). Also, dopamine may modulate working memory in an inverted U-shaped fashion, meaning that both too little or too much dopaminergic input impair working memory (Robbins, 2005). In addition, evidence has been presented that dopamine in the mPFC may modulate visuospatial attention in the 5-CSRTT in a similar manner (Granon et al, 2000).

The current findings also indicate that adolescent nicotine exposure selectively increases impulsive action measured in the 5-CSRTT, but not impulsive choice measured in the delayed reward paradigm. These findings are consistent with the notion that the two forms of impulsivity are dissociable on an anatomical and pharmacological level (Pattij and Vanderschuren, 2008; Winstanley et al, 2006a). Our neurochemical data revealed that electrically evoked dopamine release was selectively increased in slices of the $\mathrm{mPFC}$ and not Acb of periadolescent nicotine-exposed rats. Postadolescent nicotine exposure did not cause any changes in dopamine releasability in the MPFC or Acb. Considering the relationship between our behavioral and neurochemical results, accumulating data have indicated that dopamine signaling is critically involved in modulating both impulsive action and impulsive choice, but probably in different ways. For example, numerous studies indicate that the psychostimulant amphetamine increases impulsive action in the 5-CSRTT (Cole and Robbins, 1987, 1989; Harrison et al, 1997; Van Gaalen et al, 2006a), but decreases impulsive choice in delayed reward paradigms (Van Gaalen et al, 2006b; Wade et al, 2000; Winstanley et al, 2003). Recent studies have elaborated on these observations and show that primarily dopamine $\mathrm{D} 2$, and to a lesser extent dopamine $\mathrm{D} 1$, receptors appear to mediate the effects of amphetamine on impulsivity (Pattij et al, 2007; Van Gaalen et al, 2006a). In this respect, our present study shows increased dopamine releasability in the PFC following adolescent nicotine exposure, which is associated with enhanced impulsive action. Our finding that impulsive choice is not associated with such alteration of the stimulus secretion coupling process in dopamine nerve terminals is consistent with the observation that dopamine levels in the $\mathrm{MPFC}$ are not altered in rats during performance in the DRT (Winstanley et al, 2006b). Nonetheless, as yet little is known about the role of dopamine in the mPFC in impulsive action. Therefore, the causal relationship between enhanced dopamine releasability in the MPFC and impulsive action as observed here remains to be examined. Alternatively, altered - and in particular lowered - functioning of the serotonergic system has been associated with elevated impulsivity in both humans and laboratory animals (for recent reviews, see Chamberlain et al, 2006; Pattij and Vanderschuren, 2008). In this regard, it has been demonstrated recently that adolescent nicotine exposure results in long-lasting alterations in cortical serotonin functioning, an effect that was particularly pronounced in male and not female rats (Slotkin et al, 2007). Although as yet speculative, it is conceivable that changes in the serotonergic system 
may have contributed to the observed increments in impulsive action in adolescent nicotine-treated rats.

The apparent difference in altered dopamine releasability between the MPFC and Acb in response to adolescent nicotine exposure may reflect the different timing of development of these regions, as it has been shown that developing brain regions are more vulnerable to develop long-term changes in sensitivity of neurotransmitter systems (Slotkin, 2004; Slotkin et al, 2007). In particular, the mPFC is one of the last regions to develop, with a relatively late wave of neuronal loss, whereas the Acb matures earlier (Markham et al, 2007). Moreover, it has been suggested that in addition to a different timing, there might be a different developmental mechanism between $\mathrm{mPFC}$ and $\mathrm{Acb}$, as dopamine $\mathrm{D} 1$ and $\mathrm{D} 2$ receptors in the $\mathrm{mPFC}$ undergo overproduction, which subsequently leads to massive pruning during adolescence, whereas Acb dopamine receptors show overproduction at P40, but virtually no pruning occurs (Andersen et al, 2000). Also, adolescent nicotine exposure has long-term effects on morphology of a subpopulation of pyramidal neurons in the mPFC, seen as an increase in dendritic length of complex pyramidal cells (Bergstrom et al, 2008). These persistent changes induced by adolescent exposure to nicotine may be brought about by the increased induction of plasticity-associated genes, which were found to be specifically upregulated in the mPFC of animals treated with nicotine during adolescence (Schochet et al, 2005). However, there are virtually no data on the long-term effect of exogenous substances on the developing $\mathrm{MPFC}$ in terms of numbers of synapses or dopaminergic connections. Additional molecular and cellular approaches are needed to further study the putative long-term changes in the prefrontal cortical dopamine system and their role in impulsive action.

\section{Concluding Remarks}

The present data demonstrate that nicotine use during adolescence causes long-term disturbances in cognitive functioning and are consistent with a growing body of both clinical and preclinical findings. Collectively, these data strongly suggest that nicotine use during adolescence causes persistent behavioral changes that might contribute to subsequent psychopathologies. In addition, we observe long-term dopamine nerve terminal hyperresponsiveness in the mPFC upon adolescent nicotine exposure only.

Although direct comparisons of preclinical with clinical data should be interpreted with caution, the current findings stress the potential of animal models to study the long-term neurochemical and behavioral effects of adolescent nicotine exposure in more detail. Future studies should address the causal relationship between these phenomena and should provide a more in-depth analysis of the molecular and cellular mechanisms underlying the observed cognitive deficits. Most importantly, our findings imply that particularly adolescent smokers are at risk for developing long-lasting cognitive deficits.

\section{ACKNOWLEDGEMENTS}

We thank R Binnekade and H Raas $\varnothing$ for excellent technical support.

\section{DISCLOSURES/CONFLICTS OF INTERESTS}

The authors do not have any conflicts of interest to disclose.

\section{REFERENCES}

Adriani W, Deroche-Gamonet V, Le Moal M, Laviola G, Piazza PV (2006). Preexposure during or following adolescence differently affects nicotine-rewarding properties in adult rats. Psychopharmacology (Berl) 184: 382-390.

Adriani W, Spijker S, Deroche-Gamonet V, Laviola G, Le Moal M, Smit $\mathrm{AB}$ et al. (2003). Evidence for enhanced neurobehavioral vulnerability to nicotine during periadolescence in rats. J Neurosci 23: 4712-4716.

Andersen SL, Thompson AT, Rutstein M, Hostetter JC, Teicher $\mathrm{MH}$ (2000). Dopamine receptor pruning in prefrontal cortex during the periadolescent period in rats. Synapse 37: 167-169.

Arnsten AF, Li BM (2005). Neurobiology of executive functions: catecholamine influences on prefrontal cortical functions. Biol Psychiatry 57: 1377-1384.

Bergstrom HC, McDonald CG, French HT, Smith RF (2008). Continuous nicotine administration produces selective, agedependent structural alteration of pyramidal neurons from prelimbic cortex. Synapse 62: 31-39.

Bickel WK, Odum AL, Madden GJ (1999). Impulsivity and cigarette smoking: delay discounting in current, never, and ex-smokers. Psychopharmacology (Berl) 146: 447-454.

Brown RA, Lewinsohn PM, Seeley JR, Wagner EF (1996). Cigarette smoking, major depression, and other psychiatric disorders among adolescents. J Am Acad Child Adolesc Psychiatry 35: 1602-1610.

Casey BJ, Tottenham N, Liston C, Durston S (2005). Imaging the developing brain: what have we learned about cognitive development? Trends Cogn Sci 9: 104-110.

Chamberlain SR, Muller U, Robbins TW, Sahakian BJ (2006). Neuropharmacological modulation of cognition. Curr Opin Neurol 19: 607-612.

Chassin L, Presson CC, Rose JS, Sherman SJ (1996). The natural history of cigarette smoking from adolescence to adulthood: demographic predictors of continuity and change. Health Psychol 15: 478-484.

Cole BJ, Robbins TW (1987). Amphetamine impairs the discriminative performance of rats with dorsal noradrenergic bundle lesions on a 5-choice serial reaction time task: new evidence for central dopaminergic-noradrenergic interactions. Psychopharmacology (Berl) 91: 458-466.

Cole BJ, Robbins TW (1989). Effects of 6-hydroxydopamine lesions of the nucleus accumbens septi on performance of a 5-choice serial reaction time task in rats: implications for theories of selective attention and arousal. Behav Brain Res 33: 165-179.

Dalley JW, Mar AC, Economidou D, Robbins TW (2008). Neurobehavioral mechanisms of impulsivity: fronto-striatal systems and functional neurochemistry. Pharmacol Biochem Behav 90: 250-260.

Dappen A, Schwartz RH, O'Donnell R (1996). A survey of adolescent smoking patterns. J Am Board Fam Pract 9: 7-13.

Day M, Pan JB, Buckley MJ, Cronin E, Hollingsworth PR, Hirst WD et al. (2007). Differential effects of ciproxifan and nicotine on impulsivity and attention measures in the 5-choice serial reaction time test. Biochem Pharmacol 73: 1123-1134.

Diergaarde L, Pattij T, Poortvliet I, Hogenboom F, de Vries W, Schoffelmeer AN et al. (2008). Impulsive choice and impulsive action predict vulnerability to distinct stages of nicotine seeking in rats. Biol Psychiatry 63: 301-308.

Fried PA, Watkinson B, Gray R (2006). Neurocognitive consequences of cigarette smoking in young adults - a comparison with pre-drug performance. Neurotoxicol Teratol 28: 517-525. 
Godeau E, Rahav G, Hublet A (2004). Tobacco smoking. In: Currie C, Roberts C, Morgan A, Smith R, Settertobulte W, Samdal O et al (eds). Young people's health in context. Health Behaviour in School-aged Children (HBSC) study: international report from the 2001/2002 survey. World Health Organization Europe, Copenhagen, Denmark. pp 63-72.

Granon S, Passetti F, Thomas KL, Dalley JW, Everitt BJ, Robbins TW (2000). Enhanced and impaired attentional performance after infusion of D1 dopaminergic receptor agents into rat prefrontal cortex. J Neurosci 20: 1208-1215.

Grottick AJ, Higgins GA (2000). Effect of subtype selective nicotinic compounds on attention as assessed by the five-choice serial reaction time task. Behav Brain Res 117: 197-208.

Hahn B, Shoaib M, Stolerman IP (2002). Nicotine-induced enhancement of attention in the five-choice serial reaction time task: the influence of task demands. Psychopharmacology (Berl) 162: $129-137$.

Harrison AA, Everitt BJ, Robbins TW (1997). Central 5-HT depletion enhances impulsive responding without affecting the accuracy of attentional performance: interactions with dopaminergic mechanisms. Psychopharmacology (Berl) 133: 329-342.

Jacobsen LK, Krystal JH, Mencl WE, Westerveld M, Frost SJ, Pugh KR (2005). Effects of smoking and smoking abstinence on cognition in adolescent tobacco smokers. Biol Psychiatry 57: 56-66.

Jacobsen LK, Slotkin TA, Mencl WE, Frost SJ, Pugh KR (2007). Gender-specific effects of prenatal and adolescent exposure to tobacco smoke on auditory and visual attention. Neuropsychopharmacology 32: 2453-2464.

Johnson LD, O'Malley PM, Bachman JG, Schulenberg JE (2006). Monitoring the Future: National Results on Adolescent Drug Use. Overview of Key Findings 2005. NIH publication: National Institute on Drug Abuse (NIDA), Bethesda, MD. pp 278

Krishnan-Sarin S, Reynolds B, Duhig AM, Smith A, Liss T, McFetridge A et al (2007). Behavioral impulsivity predicts treatment outcome in a smoking cessation program for adolescent smokers. Drug Alcohol Depend 88: 79-82.

Markham JA, Morris JR, Juraska JM (2007). Neuron number decreases in the rat ventral, but not dorsal, medial prefrontal cortex between adolescence and adulthood. Neuroscience 144: 961-968.

Mathers M, Toumbourou JW, Catalano RF, Williams J, Patton GC (2006). Consequences of youth tobacco use: a review of prospective behavioural studies. Addiction 101: 948-958.

Miller EK, Cohen JD (2001). An integrative theory of prefrontal cortex function. Annu Rev Neurosci 24: 167-202.

Mitchell SH (1999). Measures of impulsivity in cigarette smokers and non-smokers. Psychopharmacology (Berl) 146: 455-464.

Musso F, Bettermann F, Vucurevic G, Stoeter P, Konrad A, Winterer G (2007). Smoking impacts on prefrontal attentional network function in young adult brains. Psychopharmacology (Berl) 191: 159-169.

Pattij T, Janssen MC, Vanderschuren LJ, Schoffelmeer AN, Van Gaalen MM (2007). Involvement of dopamine D1 and D2 receptors in the nucleus accumbens core and shell in inhibitory response control. Psychopharmacology (Berl) 191: 587-598.

Pattij T, Vanderschuren LJMJ (2008). The neuropharmacology of impulsive behavior. Trends Pharmacol Sci 49: 192-199.

Puumala T, Ruotsalainen S, Jakala P, Koivisto E, Riekkinen Jr P, Sirvio J (1996). Behavioral and pharmacological studies on the validation of a new animal model for attention deficit hyperactivity disorder. Neurobiol Learn Mem 66: 198-211.

Reynolds B (2004). Do high rates of cigarette consumption increase delay discounting? A cross-sectional comparison of adolescent smokers and young-adult smokers and nonsmokers. Behav Processes 67: 545-549.
Robbins TW (2005). Chemistry of the mind: neurochemical modulation of prefrontal cortical function. J Comp Neurol 493: 140-146.

Schochet TL, Kelley AE, Landry CF (2005). Differential expression of arc mRNA and other plasticity-related genes induced by nicotine in adolescent rat forebrain. Neuroscience 135 285-297.

Schoffelmeer AN, Rice KC, Jacobson AE, Van Gelderen JG, Hogenboom F, Heijna MH et al (1988). Mu-, delta- and kappa-opioid receptor-mediated inhibition of neurotransmitter release and adenylate cyclase activity in rat brain slices: studies with fentanyl isothiocyanate. Eur J Pharmacol 154: 169-178.

Skinner MD, Aubin HJ, Berlin I (2004). Impulsivity in smoking, nonsmoking, and ex-smoking alcoholics. Addict Behav 29: 973-978.

Slawecki CJ, Thorsell AK, El Khoury A, Mathe AA, Ehlers CL (2005). Increased CRF-like and NPY-like immunoreactivity in adult rats exposed to nicotine during adolescence: relation to anxiety-like and depressive-like behavior. Neuropeptides 39: 369-377.

Slotkin TA (2004). Cholinergic systems in brain development and disruption by neurotoxicants: nicotine, environmental tobacco smoke, organophosphates. Toxicol Appl Pharmacol 198: $132-151$.

Slotkin TA, MacKillop EA, Rudder CL, Ryde IT, Tate CA, Seidler FJ (2007). Permanent, sex-selective effects of prenatal or adolescent nicotine exposure, separately or sequentially, in rat brain regions: indices of cholinergic and serotonergic synaptic function, cell signaling, and neural cell number and size at 6 months of age. Neuropsychopharmacology 32: 1082-1097.

Spear LP (2000). The adolescent brain and age-related behavioral manifestations. Neurosci Biobehav Rev 24: 417-463.

Spinella M (2002). Correlations between orbitofrontal dysfunction and tobacco smoking. Addict Biol 7: 381-384.

Trauth JA, Seidler FJ, Slotkin TA (2000). Persistent and delayed behavioral changes after nicotine treatment in adolescent rats. Brain Res 880: 167-172.

Van Andel I, Rambali B, Van Amsterdam J, Wolterink G, Van Aerts LA, Vleeming W (2003). Nicotine Addiction. RIVM: Bilthoven.

Van Gaalen MM, Brueggeman RJ, Bronius PF, Schoffelmeer AN, Vanderschuren LJ (2006a). Behavioral disinhibition requires dopamine receptor activation. Psychopharmacology (Berl) 187: 73-85.

Van Gaalen MM, Van Koten R, Schoffelmeer AN, Vanderschuren LJ (2006b). Critical involvement of dopaminergic neurotransmission in impulsive decision making. Biol Psychiatry 60: 66-73.

Wade TR, de Wit H, Richards JB (2000). Effects of dopaminergic drugs on delayed reward as a measure of impulsive behavior in rats. Psychopharmacology (Berl) 150: 90-101.

Winstanley CA, Dalley JW, Theobald DE, Robbins TW (2003). Global 5-HT depletion attenuates the ability of amphetamine to decrease impulsive choice on a delay-discounting task in rats. Psychopharmacology (Berl) 170: 320-331.

Winstanley CA, Eagle DM, Robbins TW (2006a). Behavioral models of impulsivity in relation to ADHD: translation between clinical and preclinical studies. Clin Psychol Rev 26: 379-395.

Winstanley CA, Theobald DE, Dalley JW, Cardinal RN, Robbins TW (2006b). Double dissociation between serotonergic and dopaminergic modulation of medial prefrontal and orbitofrontal cortex during a test of impulsive choice. Cereb Cortex 16: 106-114. 